\title{
Advances in pleural disease
}

\author{
loannis Psallidas ${ }^{1,2}$ and Najib M. Rahman ${ }^{1,2}$
}

Affiliations: ${ }^{1}$ Oxford Centre for Respiratory Medicine and Oxford Respiratory Trials Unit, Oxford University Hospitals NHS Foundation Trust, Oxford, UK. ${ }^{2}$ Respiratory Medicine Laboratory, Nuffield Dept of Medicine, University of Oxford, Oxford, UK.

Correspondence: Ioannis Psallidas, Oxford University NHS Foundation Trust, Old Road, Churchill site, Oxford, OX3 7LE, UK. E-mail: ioannispsallidasđandm.ox.ac.uk

0 @ERSpublications

This editorial provides an update on pleural research and aims to highlight the areas that will be covered in the review series http://ow.ly/4mQDyK

The burden of pleural disease continues to increase internationally leading to significant healthcare costs and an urgent need for improving management. Pleural effusion is a common presentation for many pulmonary and systemic diseases, and it is estimated that each year 1.5 million people develop a pleural effusion in the USA and 200000-250000 in the UK [1,2]. Despite the prevalence of pleural disease, advances in research in the field have not been equal to other fields in respiratory medicine, including much rarer conditions. By way of example, talc pleurodesis (proposed in 1935) remains the standard therapy for symptomatic malignant pleural effusion in many centres [3]; very few diseases in medicine are currently managed in the same way as they were 80 years ago.

The development of pleural medicine as a subspecialty is now justified, on the basis of the increasing choice of pleural procedures that are available to the physician and the growing number of patients presenting with pleural disease [4]. It can be argued that a specialist pleural unit is now required for optimal provision of the service, as it has been shown that specialist care significantly reduces complications [5]. This also provides the best environment for providing dedicated teaching to specialty trainees and junior staff. Although a specialist pleural centre ensures effective management for patients, standardisation of practice between centres is difficult based on the lack of high-quality research that will guide clinical practice.

In the latest series in the European Respiratory Review, leading pleural specialists have provided reviews on the latest advances in pleural medicine. PsalLidas et al. [6] provide a state-of-the-art review of malignant pleural effusion from bench-to-bedside focusing on the pathogenesis, novel treatments and future directions. BHATNAGAR et al. [7] summarise the important literature relating to a number of advanced pleural interventions, including medical thoracoscopy, indwelling pleural catheters and pleural manometry. In forthcoming issues of the European Respiratory Review, benign pleural effusion, malignant pleural mesothelioma and radiological investigation of pleural disease will be covered, providing state-of-the-art advances in each area.

Research in pleural disease is now growing and large scale trials have been performed and are underway which will help answer many clinical questions. There are currently ongoing large randomised, multicentre studies aimed at: improving treatment in malignant pleural effusion and pneumothorax; understanding the pathogenesis and microbiology of pleural infection; and biomarker discovery. In parallel with clinical research, basic biological research is expected to provide much needed data on pathogenesis and mechanism, thereby suggesting novel treatments in the field. In the era of personalised medicine, translational research is anticipated to advance pleural disease through the combination of state-of-the-art laboratory research with multicentre clinical research. It is expected that the results will be applicable to everyday clinical practice.

Received: April 052016 | Accepted after revision: April 152016

Support statement: I. Psallidas is the recipient of a REPSIRE2 European Respiratory Society Fellowship (RESPIRE22015-7160). Funding information for this article has been deposited with FundRef.

Conflict of interest: None declared.

Provenance: Submitted article, peer reviewed.

Copyright CERS 2016. ERR articles are open access and distributed under the terms of the Creative Commons Attribution Non-Commercial Licence 4.0. 
References

1 Porcel JM, Light RW. Pleural effusions. Dis Mon 2013; 59: 29-57.

Bhatnagar R, Maskell N. The modern diagnosis and management of pleural effusions. BMJ 2015; 351: h4520.

Bethune N. Pleural poudrage: new technique for deliberate production of pleural adhesions as preliminary to lobectomy. J Thorac Surg 1935; 4: 251-261.

4 Hooper CE, Lee YC, Maskell NA. Setting up a specialist pleural disease service. Respirology 2010; 15: 1028-1036.

5 Havelock T, Teoh R, Laws D, et al. Pleural procedures and thoracic ultrasound: British Thoracic Society Pleural Disease Guideline 2010. Thorax 2010; 65: Suppl 2, ii61-ii76.

6 Psallidas I, Kalomenidis I, Porcel JM, et al. Malignant pleural effusion: from bench to bedside. Eur Respir Rev 2016; 25: 189-198.

7 Bhatnagar R, Corcoran JP, Maldonado F, et al. Advanced medical interventions in pleural disease. Eur Respir Rev 2016; 25: 199-213. 